\title{
ANOVA for Some Non-Normal Data by Inverting Reliability Estimators
}

\author{
Robert C. Foster \\ Bettis Atomic Power Laboratory
}

\begin{abstract}
Standard analysis of variance assumes observations are normally distributed within groups. This paper develops some analysis of variance tests for data which are Bernoulli, Poisson, exponential, or geometric distributed within groups. The tests are shown in Table 1. For natural exponential family data with conjugate priors for the distribution of means, reliability estimators directly estimate the posterior shrinkage. Using the linear posterior expectation induced by conjugate prior, a method is developed to construct an analysis of variance test by determining an appropriate transformation of a reliability estimator. The sampling distribution of the transformed reliability estimator under the assumption of group mean equality is derived to construct an appropriate test statistic. This method is used to invert the generalized KR21 estimators of Foster (2021) for some non-normal data, and it is also shown that the standard analysis of variance F-test statistic can be transformed into a consistent reliability estimator under the same assumptions. A limited simulation study shows that the inverted KR21 test has, in some scenarios, higher power than a standard analysis of variance or a generalized linear model analysis of variance.
\end{abstract}

Keywords - ANOVA, Reliability, Exponential Family, KR21, Bayesian Statistics

Robert C. Foster can be contacted at rcfoster@gmail.com 


\section{Introduction}

Traditionally, answering analysis of variance type questions about equality of group means involves an assumption of normality. The purpose of this paper is to exploit the connections between Bayesian theory, reliability, and ANOVA to show how the generalized KR21 reliability estimators of Foster (2021) can be inverted to construct distribution-specific ANOVA tests for Bernoulli, Poisson, exponential, and geometric distributed observations. The key idea is this: rather than normality of errors, the assumption is that group means follow the conjugate prior distribution. When this occurs, the linearity induced in the posterior mean allows for reliability estimators to serve as shrinkage estimators. The sampling distribution of a transformation of these reliability estimators can then be calculated under a null hypothesis of equality of means to derive an ANOVA-type test.

The historical connection between analysis of variance (ANOVA) and the classical test theory concept of reliability is strong. Hoyt (1941) famously estimated reliability using an ANOVA and derived alpha of Cronbach (1951) 10 years before Cronbach. The seminal Lord, Novick, and Birnbaum (1968) uses ANOVA to formulate estimates of some parameters in the classical test theory, and more recent work as in McGraw and Wong (1996) uses connections between intraclass correlation coefficients and analysis of variance to perform inference about the intraclass correlation coefficients. Even for dichotomous data, Feldt (1965) used an ANOVA decomposition to derive an approximate sampling distribution of formula 20 of Kuder and Richardson (1937) (KR20). The direct connection between reliability and statistical power for standard F-tests and t-tests, closely related to ANOVA, has also been highlighted in Zimmerman and Zumbo (2015) All of these methods, however, operated under the assumption of normality of observations within groups, or equivalently normality of errors.

The assumption of normality within groups is standard for ANOVA, though it is not an assumption of common classical test theory reliability estimators in general (Raykov \& Marcoulides, 2019). The effect of non-normality on the power of the ANOVA F-test has long been studied but results have been been inconclusive, though recent research suggests that the test is robust to violations of normality (Blanca, Alarcón, Arnau, Bono, \& Bendayan, 2017; Lantz, 2013), as are other linear models with Gaussian errors (Knief \& Forstmeier, 2018).

The connection between Bayesian theory and reliability is also strong. Kelley's rule of Kelley (1923) was recognized in Novick (1969) as a posterior mean under the assumption of normality using certain priors, and Novick, Jackson, and Thayer (1971) continued this effort, developing estimates of true scores from full posterior distributions, again assuming normality. Additional history and application of normal-bayes Bayesian classical test theory can be found in the eighth chapter of Levy and Mislevy (2016). Bayesian theory extends beyond normality, however. Foster (2020) showed that when sum scores follow natural exponential family distributions and the distribution of group means follow the corresponding conjugate prior, the reliability is equal to one minus the theoretical shrinkage coefficient. Foster (2021) uses this equivalence to derive a generalized version of formulas 20 and 21 of Kuder and Richardson (1937) (KR20 and KR21) for some non-normal exponential family data.

Section 2 discusses the connections between pooling as a result of Bayesian methods 
and ANOVA, focusing on the natural exponential family, conjugate priors, and the resulting posterior linearity. Section 3 shows how ANOVA tests can be derived from an invertible transformation of a reliability estimator, derives ANOVA-type hypothesis tests for some non-normal exponential family distributions by inverting the generalized KR21 reliability estimator, and shows that a function of the standard ANOVA F-test statistic is a reliability estimator for the same natural exponential family data. Section 4 conducts a simulation study comparing the inverted KR21 tests for non-normal exponential family data to a standard ANOVA and an appropriate generalize linear model ANOVA, showing that in at least some scenarios the inverted KR21 test achieves higher power than the standard ANOVA or generalized linear model ANOVA without an inflated type I error rate.

\section{Shrinkage estimation and ANOVA in the Natu- ral Exponential Family}

\subsection{ANOVA as partial versus complete pooling}

Let $y_{i j}$ be a set of observations with $i$ indexing group and $j$ indexing observation within group, and with group $i$ having mean $\theta_{i}$. In a classical test theory setting, $i$ typically indexes test item and $j$ indexes subject. Using either framework, each observation $y_{i j}$ is assumed to be identical and independent conditional on mean $\theta_{i}$. In a standard ANOVA, a linear model would typically be used for the observations $y_{i j}$.

$$
\begin{gathered}
y_{i j}=\theta_{i}+\epsilon_{i j} \\
\epsilon_{i j} \stackrel{i i d}{\sim} N\left(0, \sigma^{2}\right)
\end{gathered}
$$

In the linear model, the means $\theta_{i}$ are assumed fixed and the errors $\epsilon_{i j}$ are random. If the null hypothesis of equality of means is rejected in the ANOVA hypothesis test, the mean for each group is estimated using only the observations within each group, with no sharing of information between groups. If the null hypothesis of equality of means can not be rejected in the ANOVA hypothesis test, each mean is estimated as the same global mean of all observations.

Consider instead a hierarchical model for the observations, where the means themselves follow some distribution $g\left(\theta_{i}\right)$.

$$
\begin{aligned}
y_{i j} & \sim f\left(y_{i j} \mid \theta_{i}\right) \\
\theta_{i} & \sim g\left(\theta_{i}\right)
\end{aligned}
$$

Bayesian methods are a natural fit for hierarchical models, and produce "pooled" estimates of means $\theta_{i}$ which combine information both within groups and between the groups. The use of pooled estimates of group means $\theta_{i}$ can be thought of as a compromise between the zero pooling that occurs when the ANOVA hypothesis test rejects equality of means and the complete pooling which occurs when the ANOVA hypothesis fails to reject equality of means (Gelman \& Hill, 2006). 
The exact amount of pooling depends on the distribution of the means $g\left(\theta_{i}\right)$ and does not typically have a closed-form expression for arbitrary distributions $g\left(\theta_{i}\right)$. However, the pooling amount is known for specific classes of distributions. One such class of distributions is the conjugate priors available when observations $y_{i j}$ can be said to follow an exponential family distribution.

\subsection{Reliability in the Natural Exponential Family with Con- jugate Priors}

The natural exponential family is a subset of the exponential family and a class of distributions which includes the normal distribution, the Bernoulli, the Poisson, the negative binomial, and the exponential, which are among the most commonly used non-normal distributions in health, educational, and social sciences (Bono, Blanca, Arnau, \& Gómez-Benito, 2017). Properties of the natural exponential family, often called the one-parameter exponential family, are discussed in detail in Morris (1982) and Morris (1983). The properties of equal difficulty exponential family test data in classical test theory, including reliability, are discussed in Foster (2020). This paper will state without proof only which properties of the exponential family are necessary.

Let observation $y_{i j}$ be a draw from a random variable $Y_{i j}$ which follows a natural exponential family generator distribution. These draws are identical and independent conditional on group mean $\theta_{i}$, which is assumed to be the same for all observations within a group, equivalent to the assumption of equal difficulty test items (though $\theta_{i}$ may differ between groups). Generator distributions generate a larger family through summation (convolution). Some examples of generator distributions are Bernoulli distributions which generate the binomial, Poisson distributions which generate the Poisson, exponential distributions which generate the gamma, and geometric distributions which generate the negative binomial. A key feature of these distributions is that the variance is a polynomial function of the mean $\theta_{i}$.

$$
\operatorname{Var}\left(Y_{i j} \mid \theta_{i}\right)=V\left(\theta_{i}\right)=v_{0}+v_{1} \theta_{i}+v_{2} \theta_{i}^{2}+\ldots
$$

For example, data which is Bernoulli distributed with mean proportion $\theta_{i}$ has variance $\theta_{i}\left(1-\theta_{i}\right)=\theta_{i}-\theta_{i}^{2}$, so it fits Equation (1) with $v_{1}=1, v_{2}=-1$, and all other terms 0 . The exponential distribution with mean $\theta_{i}$ has variance $\theta_{i}^{2}$, so it fits with $v_{2}=1$ and all other terms 0.

Let the random variable $X_{i}$ be defined as the sum over all $n$ observations for each group.

$$
X_{i}=\sum_{j=1}^{n} Y_{i j}
$$

The natural exponential family is closed under convolution of these generator distributions (Morris, 1983) when identical and independent, and so the sum $x_{i}$ is a member of the same natural exponential family. Using the law of total expectation, the conditional and unconditional means of the $x_{i}$ are 


$$
\begin{aligned}
E\left[X_{i} \mid \theta_{i}\right] & =n \theta_{i} \\
E\left[X_{i}\right] & =n \mu
\end{aligned}
$$

and using the law of total variance, the conditional and unconditional means of the $x_{i}$ are

$$
\begin{array}{r}
\operatorname{Var}\left(X_{i} \mid \theta_{i}\right)=n V\left(\theta_{i}\right) \\
\operatorname{Var}\left(X_{i}\right)=n E\left[V\left(\theta_{i}\right)\right]+n^{2} \operatorname{Var}\left(\theta_{i}\right)
\end{array}
$$

The conditional and unconditional means of individual observations $y_{i j}$ can be found by using $n=1$ in Equations (2) and (3).

All exponential family distributions are guaranteed to have conjugate priors (Morris, 1983). Assume that the distribution of group means $g\left(\theta_{i}\right)$ follows the conjugate prior distribution $g\left(\theta_{i} \mid \mu, M\right)$, where $\mu$ and $M$ are defined as

$$
\begin{array}{r}
\mu=E\left[\theta_{i}\right] \\
M=E\left[V\left(\theta_{i}\right)\right] / \operatorname{Var}\left(\theta_{i}\right)
\end{array}
$$

and where $V\left(\theta_{i}\right)$ is the variance function of the $y_{i j}$ given in Equation (1).

Diaconis and Ylvisaker (1979) showed that when the exponential family is assumed for observations, a linear posterior expectation occurs if and only if the conjugate prior is also assumed for means. That is, if the estimate $\hat{\theta}_{i}$ is taken as the mean of the posterior distribution of $\theta_{i}$

$$
\hat{\theta}_{i}=E\left[\theta_{i} \mid \tilde{y}\right]
$$

then $\hat{\theta}_{i}$ is a linear function of the data

$$
\hat{\theta}_{i}=(1-B) \bar{y}_{i}+B \mu
$$

if and only if the distribution $g\left(\theta_{i} \mid \mu, M\right)$ is the conjugate prior to the chosen natural exponential family distribution of the $y_{i j}$. The parameter $B$ is the shrinkage coefficient, equal to

$$
B=\frac{M}{M+n}
$$

Foster (2020) showed that when the natural exponential family is assumed for independent and identical observations $y_{i j}$ given group or subject mean $\theta_{i}$, and $\theta_{i}$ follows the corresponding conjugate prior distribution, the shrinkage coefficient $B$ is equal to $1-\rho$, where $\rho$ is the reliability. Equation (5) can thus be written as a function of the reliability $\rho$.

$$
\hat{\theta}_{i}=\rho \bar{y}_{i}+(1-\rho) \mu
$$

where

$$
\rho=\frac{n}{M+n}
$$


Equations (4) and (6) using $\bar{y}$ as an estimator for $\mu$ and any estimator of reliability $\hat{\rho}$ can be seen as a form of Kelley's rule of Kelley (1923). The formulation in $\rho$ is important because estimators such as Cronbach's alpha and Kuder-Richardson formulas 20 and 21, traditionally thought of as estimators of reliability $\rho$, can also be used as estimators of the amount of posterior shrinkage.

When does the reliability $\rho=0$ ? Assuming the test length $n$ is nonzero in Equation (7), 0 is the limiting value of $\rho$ as $M$ tends towards infinity. The theoretical $M=\infty$ occurs when $E\left[V\left(\theta_{i}\right)\right] / \operatorname{Var}\left(\theta_{i}\right)=\infty$, or when $\operatorname{Var}\left(\theta_{i}\right)=0$. A value of $\rho=0$ therefore implies $g\left(\theta_{i} \mid \mu, M\right)$ is a degenerate distribution which takes on only a single value $\mu$ with probability $1, P\left(\theta_{i}=\mu\right)=1$, and that all group means $\theta_{i}$ are equal to a common mean $\mu$.

\section{Hypothesis Tests as Inverted Reliability Estima- tors}

When the population reliability $\rho=0$ in Equation (6), the estimate of each group mean $\theta_{i}$ is simply the common mean $\mu$. When $\rho>0$, the estimate of each group mean is a unique linear combination of the estimated group and common means. Hence, a hypothesis test of $\rho=0$ is a test of equality of means. Given a reliability estimator $\hat{\rho}$, the sampling distribution of a transformation of $\hat{\rho}$ can be used to test $\rho=0$ versus $\rho>0$, which will be referred to as an inverted reliability estimator test. This idea been expressed for the normal-normal model, which assumes normality for both observations $y_{i j}$ and group means $\theta_{i}$, for a shrinkage estimator $\hat{B}$ in Casella (1985). While Casella (1985) made an explicit assumption of normality, there is nothing in the core idea of using the sampling distribution of an estimator to obtain a hypothesis test which forces normality. As Raykov and Marcoulides (2019) states, common reliability estimators such as Cronbach's alpha make no assumption of normality. The idea of inverting a reliability or shrinkage estimator can be expanded to estimators which make explicit assumptions of non-normality.

\subsection{The Method of Inverting a Shrinkage Estimator}

The method of inverting a reliability estimator is as follows: suppose that observations $y_{i j}$ identically and independently follow some natural exponential family generator distribution given group mean $\theta_{i}$, and suppose that the distribution of $\theta_{i}$ follows the corresponding conjugate prior $g\left(\theta_{i} \mid \mu, M\right)$. The estimate of group mean $\hat{\theta}_{i}$ given by the posterior group mean is equal to

$$
\hat{\theta}_{i}=\rho \bar{y}_{i}+(1-\rho) \mu
$$

where $\rho$ is the reliability, as in Equation (6). Alternatively, it may be formulated with the shrinkage coefficient $B=1-\rho$, as in Equation (4). It is not necessary to know or estimate $\mu$. The null hypothesis and alternative hypotheses are of the form 


$$
\begin{gathered}
H_{0}: \theta_{i}=\mu \text { for all } i \\
H_{A}: \theta_{i} \text { follow some random distribution }
\end{gathered}
$$

This set of null and alternative hypotheses can also be though of as a test that $g\left(\theta_{i} \mid \mu, M\right)$ is a degenerate distribution that takes on a single value $\mu$ with probability 1 versus that it is a non-degenerate distribution, or that $\rho=0$ versus $\rho>0$ where $\rho$ is the reliability, or that $M=\infty$ versus $M$ is finite where, $M$ is the parameter in the distribution $g\left(\theta_{i} \mid \mu, M\right)$.

Let $\hat{\rho}$ be any consistent estimator of the reliability $\rho$ and let $T(\hat{\rho})$ be any invertible transformation of the reliability estimator $\hat{\rho}$ such that $T(\hat{\rho})$ follows a known distribution when the null hypothesis is true.

$$
T(\hat{\rho}) \sim W \text { when } \theta_{i}=\mu \text { for all } i
$$

Then $T(\hat{\rho})$ functions as a test statistic for the null hypothesis that all group means are equal. The p-value for testing this hypothesis is constructed as the probability of the test statistic exceeding in magnitude a quantile corresponding to some critical value $\alpha$ from the reference distribution $W$. If the p-value is less than $\alpha$, the null hypothesis is rejected.

Note that this method of inverting reliability estimators may just as easily be applied to estimators $\hat{B}$ of the shrinkage coefficient $B$ given in Equation (5), similarly to Casella (1985). Shrinkage estimators are more commonly associated with empirical Bayesian methods.

\subsection{Inverting the Generalized KR-21 Estimator}

The generalized KR-21 estimators of Foster (2021) are ideal for inversion in this manner. The estimators make similar assumptions as an ANOVA: that each observation $y_{i j}$ is identical and independent given group mean $\theta_{i}$, equivalent to assuming equal difficulty for all test items in the test reliability setting. Rather than Gaussian errors, however, the estimators also explicitly assume that the observations $y_{i j}$ are non-normal within groups, and that the distribution of group means follows the corresponding conjugate prior, exactly as in Section 3.1. The generalized KR21 is available when observations $y_{i j}$ follow one of the normal distribution (with known variance $\sigma^{2}$ ), the Bernoulli, the Poisson, the exponential, the geometric, or the hyperbolic secant distribution.

For these distributions, the generalized KR-21 estimator is:

$$
K R 21=\frac{n}{n+v_{2}}\left(1-\frac{n V\left(\frac{1}{n} \bar{x}\right)}{s_{x}^{2}}\right)
$$

where $V\left(\frac{1}{n} \bar{x}\right)$ is the variance function of the exponential family distribution of the observations $y_{i j}$ as in Equation (1). Foster (2021) showed that the estimator in Equation (8) is a consistent estimator of $\rho$. Note that $s_{x}^{2}$ is guaranteed to be nonnegative, though it can be equal to zero. The variance function $V\left(\theta_{i}\right)$ is strictly positive so long as the distribution of $\theta_{i}$ is not degenerate (Morris, 1982). Thus, the quantity $n V\left(\frac{1}{n} \bar{x}\right) / s_{x}^{2}$ is guaranteed to be positive. The supremum of the values that the generalized KR21 estimator may obtain is therefore $n /\left(n+v_{2}\right)$, though it may occasionally produce a negative estimated reliability. 
Equation (8) gives an estimator $\hat{\rho}$ of the reliability as

$$
\hat{\rho}=\frac{n}{n+v_{2}}\left(1-\frac{n V\left(\frac{1}{n} \bar{x}\right)}{s_{x}^{2}}\right)
$$

Define the transformation $T(\hat{\rho})$ as

$$
T(\hat{\rho})=\frac{k-1}{1-\frac{n+v_{2}}{n} \hat{\rho}}
$$

Equation (9) is a rational function with an asymptote at $\hat{\rho}=n /\left(n+v_{2}\right)$. As previously stated, $n /\left(n+v_{2}\right)$ is the supremum of values that $\hat{\rho}$ can obtain. Hence, the transformation is invertible and positive over the image of $\hat{\rho}$. When the transformation in Equation (9) is applied to the estimator in Equation (9), the result is

$$
T(\hat{\rho})=\frac{\sum_{i=1}^{k}\left(x_{i}-\bar{x}\right)^{2}}{n V\left(\frac{1}{n} \bar{x}\right)}=\frac{(k-1) s_{x}^{2}}{n V\left(\frac{1}{n} \bar{x}\right)}
$$

Under the null hypothesis, it is assumed that $\theta_{i}=\mu$ for all groups. Because $x_{i}$ is defined as the sum over independent and identical observations $y_{i j}$ in each group, and each $y_{i j}$ has finite mean and variance by the exponential family assumption, the distribution of $x_{i}$ can be approximated by a normal distribution when $n$ is large by the central limit theorem. When the distribution of $g\left(\theta_{i} \mid \mu, M\right)$ is a degenerate distribution that takes on $\mu$ with probability 1 under the null hypothesis, both the conditional and unconditional mean of $x_{i}$ is equal to $n \mu$ in Equation (2) and the conditional and unconditional variance is equal to $n V(\mu)$ in Equation (3), as $\operatorname{Var}\left(\theta_{i}\right)=0$ and $n E\left[V\left(\theta_{i}\right)\right]=n E[V(\mu)]=n V(\mu)$.

$$
x_{i} \sim N(n \mu, n V(\mu))
$$

Hence, $E\left[\frac{1}{n} \bar{x}\right]=\frac{1}{n}(n \mu)=\mu$ and $n V\left(\frac{1}{n} \bar{x}\right)$ is a consistent estimator for the variance $n V(\mu)$ by the continuous mapping theorem and the law of large numbers. When the number of observations $n$ within each group is large, the square root of each term of the the test statistic in Equation (10) can be approximated by a standard normal distribution.

$$
\frac{x_{i}-\bar{x}}{\sqrt{n V\left(\frac{1}{n} \bar{x}\right)}} \sim N(0,1)
$$

The sum of $k$ squared standard normal distributions is approximately chi-squared with degrees of freedom $k-1$ (Casella \& Berger, 2002). Thus, when the number of observations $n$ within each group is large, the distribution of the test statistic in Equation (10) can be approximated as 


$$
\frac{\sum_{i=1}^{k}\left(x_{i}-\bar{x}\right)^{2}}{n V\left(\frac{1}{n} \bar{x}\right)} \sim \chi_{k-1}^{2}
$$

The p-value for the test is $P\left(\chi_{k-1}^{2}>T(\hat{\rho})\right)$.

A list of test statistics given by Equation (10) for dichotomous (Bernoulli distributed) data, Poisson data, exponential data, and geometric data is shown in Table 1. To be clear, several of these tests have been previously proposed, but typically in different contexts than ANOVA. The Poisson test statistic in table 1 was originally proposed in Fisher (1925) as a test of equality of variances for the Poisson distribution. A test of equality of Poisson variances is a test of equality of means because the mean is equal to the variance for a Poisson distribution. The test for the dichotomous data is exactly the test for nonzero reliability as estimated by KR21 in Keats (1964), which served as the primary inspiration for this paper. The author has been unable to find the exact formulas given in Table 1 for exponential and geometric data in previous research, but given the extensive literature of reliability, analysis of variance, and chi-squared goodness of fit tests, it is likely that they have been derived under alternate contexts. The usage of these formulas for ANOVA, however, appears to the author to be novel.

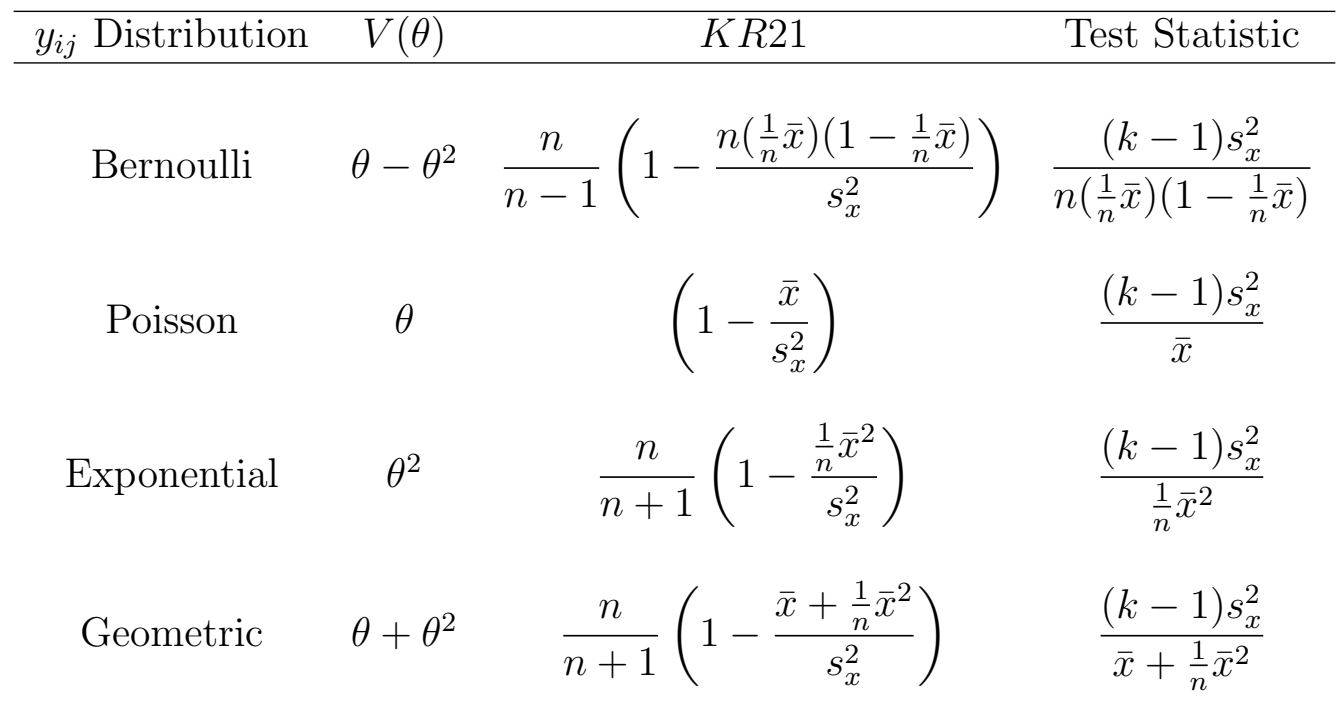

Table 1: The generalized KR21 estimator and corresponding test statistic for Bernoulli, Poisson, Exponential, and geometric data. The number of groups is $k$ and the number of observations within each group is $n$, assumed to be equal for all groups. The sum of all observations $y_{i j}$ in group $i$ is $x_{i}$. The reference distribution for each test statistic is a $\chi_{k-1}^{2}$ for large $n$ by the central limit theorem, though it will not be exact for small $n$. The Poisson KR21 estimator was first derived in Allison (1978), the binomial estimator is the classic KR21 of Kuder and Richardson (1937), and the others were derived in Foster (2021).

Note that it is also possible to invert the KR-20 estimator using Equation (9) to 
obtain a test; however, caution must be taken with the reference distribution of the test statistic. Simulations appear to indicate that inverting the traditional KR-20 for dichotomous data rather than KR-21, for example, yields a $\chi_{k-2}^{2}$ reference distribution.

\subsection{The ANOVA Test Statistic as an Inverted Reliability Es- timator}

A natural question to ask is how the traditional ANOVA hypothesis test fits within the framework of inverted reliability estimators. The answer is that the standard ANOVA Ftest statistic can be transformed into a consistent estimator of reliability under the same assumptions as Section 2: that observations $y_{i j}$ are independent and identical observations from some natural exponential generator distribution conditional on group mean $\theta_{i}$, and that the distributions of group means $\theta_{i}$ follow the corresponding conjugate prior distribution.

Consider the standard ANOVA test statistic, given by the ratio of mean squares for treatment to mean squares for error. When all groups have equal sample sizes $n$, the test statistic is

$$
F=\frac{M S T r t}{M S E}=\frac{\frac{1}{k-1} \sum_{i=1}^{k} n\left(\bar{y}_{i}-\bar{y}\right)^{2}}{\frac{1}{k n-k} \sum_{i=1}^{k}(n-1) s_{i}^{2}}
$$

The numerator, MSTrt, can be written as

$\frac{1}{k-1} \sum_{i=1}^{k} n\left(\bar{y}_{i}-\bar{y}\right)^{2}=\frac{1}{n(k-1)} \sum_{i=1}^{k}\left(\sum_{j=1}^{n} y_{i j}-\frac{1}{k} \sum_{i=1}^{k} \sum_{j=1}^{n} y_{i j}\right)^{2}=\frac{1}{n(k-1)} \sum_{i=1}^{k}\left(x_{i}-\bar{x}\right)^{2}=\frac{1}{n} s_{x}^{2}$

$M S r t$ is $1 / n$ times the sample variance of the $x_{i}$. Hence, the expected value of MSTrt in the exponential family is

$$
E[M S T r t]=E\left[\frac{1}{n} s_{x}^{2}\right]=\frac{1}{n} \operatorname{Var}\left(x_{i}\right)
$$

Using Equation (3), the unconditional variance is

$$
\frac{1}{n} \operatorname{Var}\left(x_{i}\right)=E\left[V\left(\theta_{i}\right)\right]+n \operatorname{Var}\left(\theta_{i}\right)
$$

For $M S E$, when the sample sizes are balanced and each group size is equal to $n$, it reduces to the average of group variances $s_{i}^{2}$. 


$$
\frac{1}{k n-k} \sum_{i=1}^{k}(n-1) s_{i}^{2}=\frac{n-1}{k(n-1)} \sum_{i=1}^{k} s_{i}^{2}=\frac{1}{k} \sum_{i=1}^{k} s_{i}^{2}
$$

The expected value of $M S E$ is

$$
E[M S E]=E\left[\frac{1}{k} \sum_{i=1}^{k} s_{i}^{2}\right]=\frac{1}{k} \sum_{i=1}^{k} E\left[s_{i}^{2}\right]
$$

Using the law of total expectation, this becomes

$$
\frac{1}{k} \sum_{i=1}^{k} E\left[s_{i}^{2}\right]=\frac{1}{k} \sum_{i=1}^{k} E\left[E\left[s_{i}^{2} \mid \theta_{i}\right]\right]=\frac{1}{k} \sum_{i=1}^{k} E\left[V\left(\theta_{i}\right)\right]=\frac{1}{k}\left(k E\left[V\left(\theta_{i}\right)\right]\right)=E\left[V\left(\theta_{i}\right)\right]
$$

Hence, the ratio of expected values for the $\mathrm{F}$ test statistic is

$$
\frac{E[M S T r t]}{E[M S E]}=\frac{E\left[V\left(\theta_{i}\right)\right]+n \operatorname{Var}\left(\theta_{i}\right)}{E\left[V\left(\theta_{i}\right)\right]}=\frac{M+n}{M}=\frac{1}{1-\rho}
$$

The law of large numbers shows that, for the well-behaved natural exponential family, each of the numerator and denominator of the $\mathrm{F}$ test statistic are converging to their expectations, the ratio of which gives the inverse of one minus the reliability $\rho$. By Slutsky's theorem, the F-test statistic is consistent for $1 /(1-\rho)$. By the continuous mapping theorem, a consistent estimator for the reliability is therefore

$$
\hat{\rho}=1-\frac{1}{F}
$$

where $\mathrm{F}$ is the standard test statistic for the ANOVA test.

Equation (12) is the same estimator of reliability for $\operatorname{ICC}(k)$ in the one-way ANOVA model derived in McGraw and Wong (1996). The derivation McGraw and Wong (1996), however, was made under the traditional assumptions of normality. With equal sample sizes $n$ for each group, Equation (12) is a consistent estimator of reliability under the following assumptions:

1. Each observation $y_{i j}$ independently and identically follows the same natural exponential family generator distribution conditional on group mean $\theta_{i}$

2. The distribution of group means $g\left(\theta_{i}\right)$ follows the conjugate prior to the natural exponential family distribution of the $y_{i j}$ 
Though the ANOVA F-test statistic can be inverted to estimate reliability for some non-normal observations, the standard ANOVA hypothesis test is still only valid under the assumption of normality. This is because normality is used to derive the F reference distribution which is used to determine p-values and statistical significance. The distribution of the test statistic under non-normality is not $\mathrm{F}$ distributed. However, note that the numerator of the $\mathrm{F}$ test statistic can be written as a function of the sample variance of the distribution of sum scores $x_{i}$. The distribution of $x_{i}$ is approximately normal for large numbers of observations $n$ by the central limit theorem. Similarly, the denominator is a sum of identical and independent random variables when all means $\theta_{i}$ are equal because the mean-variance relation implies that equal group means yields equal group variances. Hence, the distribution of the sum of squared errors also approaches a normal as $n$ or $k$ increases, which is the same limiting distribution of the $\chi_{n k-k}^{2}$ distribution derived under the assumption of normality in traditional ANOVA. Though only suggestion and not proof, this may serve to partly explain why ANOVA appears to perform near nominally for some non-normal data the sample size within groups $n$ is large.

\section{Simulation Study}

Section 3.2 derived some distribution-specific ANOVA tests by inverting the generalized KR21 estimators and Section 3.3 showed that the standard ANOVA test statistic can be seen as an inverted reliability estimator for the natural exponential family, though the reference $F$ distribution for the test statistic is still only applicable to normally distributed data. But why should the tests of Section 3.2 should be preferred over standard ANOVA, or over other distribution-specific methods such as generalized linear models? A simulation study shows that in at least some situations, the inverted KR21 tests of Section 3.2 provide superior power over both a standard ANOVA test and generalized linear model while not inflating the type I error rate.

\subsection{Methodology}

The purpose of this section is to simulate non-normal data for which some type of ANOVA test would typically be considered and compare the properties of the inverted KR21 tests obtained in Section 3.2 to more standard estimators. Data for which an ANOVA test is considered appropriate typically consists of multiple groups of data with observations within each group being identical and independent conditional on group mean $\theta_{i}$. The goal of an ANOVA test is to determine if $\theta_{i}$ is equal to some constant value $\mu$ for all groups or if at least one $\theta_{i}$ differs from the rest.

The simulation study must therefore have as parameters the non-normal distribution of individual observations $y_{i j}$, the group means $\theta_{i}$, the number of observations $n$ within each group, and the number of groups $k$. For this simulation study, observations $y_{i j}$ were simulated in the following manner:

1. Choose the natural exponential family generator distribution for the distribution of the observations $y_{i j}$ given group mean $\theta_{i}$. 
2. Choose the number of groups $\mathrm{k}$, the number of observations $n$ within each group, and the common mean $\mu$ for each group.

3. Choose the amount $\delta$ added to a single group mean.

4. For the first group, simulate $n$ values from the distribution of $y_{i j}$ with mean $\theta_{i}=\mu+\delta$. For groups 2 to $k$, simulate $n$ values from the distribution of $y_{i j}$ with mean $\theta_{i}=\mu$.

For example, a single simulated data set using the exponential distribution with $k=5$ groups of $n=30$ observations each, $\mu=1$, and $\delta=0.5$ would simulate $n=30$ observations for the first group from an exponential distribution with mean $\theta_{1}=\mu+\delta=1.5$ and $n=30$ observations for each of the final four groups from an exponential distribution with mean $\theta_{i}=\mu=1$.

The method of deriving an ANOVA test by inverting the reliability estimator in Section 3 explicitly makes the assumption that the distribution of group means $\theta_{i}$ follows the conjugate prior to the distribution of the observations $y_{i j}$. However, the conjugate prior was not used in the simulation process. The goal of this simulation strategy is to show that the ANOVA tests for non-normal data of Section 3.2 are still valid in the traditional ANOVA framework that does not assign distributions to group means and that the tests are robust to violations of the assumption that the distribution of means follows the conjugate prior distribution. The results of simulation studies may be overly optimistic when the same model is used for both data simulation and estimation, and the use of a different model for data simulation should help to avoid this issue.

For this simulation study, the possible non-normal distributions of the observations $y_{i j}$ are Bernoulli, Poisson, exponential, and geometric. Both the inverted KR21 test of Section 3.2 given in Table 1 and a generalized linear model is available for each distribution. For the number of observations $n$ within each group, the values $n=10$ and $n=30$ were used. These were picked to compare a small sample size to a moderate sample size. For large $n$, all methods should produce approximately the same results. For small sample sizes, the inverted KR21 test depends on asymptotic normality of the sum of observations in each group $x_{i}$ and so should not have nominal coverage. The purpose of the small and moderate sample sizes is to show convergence to nominal values as the number of observations $n$ within each group increases and to determine properties of any tests in a scenario that it should not be expected to perform nominally. For the number of groups, the values of $k=2,5$, and 10 were used. These were chosen with $k=2$ as the smallest number of possible groups that can be compares using an ANOVA test, and the others simply increasing the number of groups to determine the effect. For the common group mean $\mu$, the value of $\mu=0.25$ was chosen for Bernoulli $y_{i j}, \mu=1$ for Poisson $y_{i j}, \mu=1$ for exponential $y_{i j}$, and $\mu=1.01$ for geometric $y_{i j}$. These values were chosen as reasonable values which worked well in practice, and because the distributions of the sums $x_{i}$ are still skewed for $n=10$, though such skew is often significantly lessened by $n=30$. The inverted KR21 tests of Section 3.2 are based on the convergence of these sums $x_{i}$ to a normal distribution by the central limit theorem, and a skew ensures that any positive results are not simply because the summed data nearly follow a normal distribution even if the individual observations do not. These values of $\mu$ were used in all $n$ and $k$ combinations for a given distribution. Lastly for $\delta$, the value $\delta=0$ was used to determine the type I error rate and a series of twenty equally spaced $\delta$ values 
between 0 and 0.5 for Bernoulli $y_{i j}$, between 0 and 2 for Poisson $y_{i j}$, between 0 and 2 for exponential $y_{i j}$, and between 0 and 2.5 for geometric $y_{i j}$ were used. This yields a total of 21 possible $\delta$ values for each $n$ and $k$ pair. These values were chosen so that for the largest number of groups and observations $k=10$ and $n=30$ the power of each test at the largest $\delta$ value was very nearly equal to 1 .

Data that follows a natural exponential family such as the binomial, Poisson, or negative binomial are often modeled using a generalized linear model (GLM) when an ANOVA is needed (Nelder \& Wedderburn, 1972). Hence, for the simulated data, three possible ANOVA tests are considered:

1. A standard one-way ANOVA F test

2. A GLM ANOVA test after fitting the appropriate generalized linear model for the exponential family distribution

3. The inverted KR21 ANOVA test for the exponential family distribution, given in Table 1 of Section 3.2

For the generalized linear models, the dispersion parameter $\phi$ was fixed at $\phi=1$ in all cases. For the negative binomial generalized linear model, which traditionally is taken to have variance function $V(\theta)=\theta+\left(\theta^{2} / \kappa\right)$, the parameter $\kappa$ was also taken to be 1 . Presumably, if an experimenter knew the distributions of the observations $y_{i j}$ exactly as independent and identical Bernoulli, Poisson, exponential, or geometric distributions, the dispersion and other parameters would be known as well. The canonical link function was used for all GLMs. Fitting of each GLM was performed using the glm.fit() function in the $\mathbf{R}$ program and in the mass package for negative binomial generalized linear models.

The response is the proportion of times each test rejects the null hypothesis of equality of group means. When $\delta>0$, this is the power of the test. When $\delta=0$ and the null hypothesis is true, this is the type I error rate.

A total of four million simulated data sets were simulated at each for each combination of parameters. This ensures that the width of a $95 \%$ confidence interval for the power of the test at any given set of parameters is less than or equal to $2 \times 1.96 \times$ $\sqrt{0.5(1-0.5) / 4000000}=0.0098$, or less than 0.001 , and so three decimal places of accuracy in reported results is justified. A $95 \%$ interval for each estimated power is simply the rounding error, and often much less for estimated powers not near 0.5.

\subsection{Results}

Figure 1 shows the results for Bernoulli distributed data in two plots. The top plot shows the difference in power between the inverted KR21 test and standard ANOVA for Bernoulli observations plotted as a function of the first group mean difference $\theta$ for all combinations of $n$ and $k$. The bottom plot shows the same between the inverted KR21 test and a binomial GLM ANOVA. Table 2 gives the exact powers of each test for the specific values of $\delta=0, \delta=0.25$, and $\delta=0.5$ that are used to calculate the differences in Figure 1. For

$k=2$, the test statistic from the standard ANOVA and the inverted KR21 test are very close (though not equal), and so the power of each test in Table 2 is identical up to three 
decimal places due to the similarity between the $F_{1, n-2}$ and $\chi_{1}^{2}$ distributions, though the power is not guaranteed to be equal in all cases. The results show that the power is close for all three tests, with the GLM ANOVA typically having the highest power. However, the GLM ANOVA also has an inflated type I error rate, reaching up to 0.092 when $k=10$ and $n=10$, when the power for the ANOVA and inverted KR21 tests are 0.050 and 0.045, respectively. In general, the inverted KR21 test always has power less than or equal to that of the standard ANOVA, which in turn has smaller power than the GLM ANOVA when $n=10$ but larger power when $n=30$ and $k>2$.

Figure 2 shows plots of the difference in power between the inverted KR21 test and standard ANOVA for Poisson distributed observations, and between the inverted KR21 test and a GLM ANOVA, again as a function of first group mean difference $\theta$. Table 3 gives exact powers for $\delta=0, \delta=1$, and $\delta=2$. The results show that the inverted KR21 test is nearly universally superior to the standard ANOVA in terms of power, and usually superior to the Poisson GLM. The type I error rate is nearly nominal for all three, though the Poisson GLM's type I error rate is very slightly inflated.

Similarly, Figure 3 shows plots of the difference in power between the inverted KR21 test and standard ANOVA for exponential distributed observations, and between the inverted KR21 test and a GLM ANOVA, as a function of first group mean difference $\theta$. Table 3 gives exact powers of each test for $\delta=0, \delta=1$, and $\delta=2$. And similarly, the inverted KR21 is nearly universally superior to a standard ANOVA. It is only superior to the GLM ANOVA when the number of groups $k$ is larger than 2, however. The increase in power is sizeable, however - up to $12 \%$ in magnitude increase in power over a standard ANOVA and up to $8 \%$ increase in magnitude over a GLM ANOVA. For this data, the inverted KR21 test and the standard ANOVA have slightly depressed type I error rates when $n$ and $k$ are small, while the GLM is closer to the nominal $\alpha=0.05$.

Lastly, Figure 4 shows plots of the difference in power between the inverted KR21 test and standard ANOVA for geometric distributed observations, and between the inverted KR21 test and a GLM ANOVA, as a function of first group mean difference $\theta$. Table 5 gives exact powers of each test for $\delta=0, \delta=1.25$, and $\delta=2.5$. Once again, the inverted KR21 test is nearly universally superior to the standard ANOVA, and appears to be superior to the GLM ANOVA for $k>2$. The power increase is significant, with an increase of up to $10 \%$ magnitude power of the inverted KR21 test over the standard ANOVA, and up to 8\% over the GLM ANOVA. The type I error rates are slightly deflated for the inverted KR21 and standard ANOVA when $n$ and $k$ are small, and in the GLM ANOVA type I error rate is slightly inflated in the same scenarios, but in all cases it is nearly nominal and within a $1 \%$ magnitude difference.

As discussed for the generalized KR21 estimator in Foster (2021), one possible reason for the improved performance of the inverted KR21 test over standard ANOVA is that it is estimating a variance $n V(\mu)$ by using a sample mean in combination with the mean-variance relationship for the distribution $n V\left(\frac{1}{n} \bar{x}\right)$ rather than the sample variance $s^{2}$. Sample means converge at a faster rate than sample variances, and so the test statistic can be thought of as more efficient. A second reason is the issue of bias. The test statistic given in Equation (10) treats the sample quantity $n V\left(\frac{1}{n} \bar{x}\right)$ as the population variance to derive the $\chi_{k-1}^{2}$ reference distribution; however, it is still an estimate, and so will affect the test statistic probabilistically. The function $f(x)=1 / x$ is convex, and and so $E\left[1 /\left(n V\left(\frac{1}{n} \bar{x}\right)\right]>1 / E\left[n V\left(\frac{1}{n} \bar{x}\right)\right]\right.$ 
by Jensen's inequality. However, Jensen's inequality also applies to the quantity $n V\left(\frac{1}{n} \bar{x}\right)$. For Bernoulli distributed data, the variance function $V\left(\theta_{i}\right)=\theta_{i}-\theta_{i}^{2}$ is concave, and so $E\left[V\left(\frac{1}{n} \bar{x}\right)\right]<V\left(E\left[\frac{1}{n} \bar{x}\right]\right)=V(\mu)$. Then $E\left[1 /\left(n V\left(\frac{1}{n} \bar{x}\right)\right)\right]>1 / E\left[n V\left(\frac{1}{n} \bar{x}\right)\right]>1 /(n V(\mu))$, making the bias worse. In contrast, for geometric and exponential data, the variance function $V\left(\theta_{i}\right)$ is convex, and so $E\left[n V\left(\frac{1}{n} \bar{x}\right)\right]>n V(\mu)$ and $1 / E\left[n V\left(\frac{1}{n} \bar{x}\right)\right]<1 /(n V(\mu))$ by Jensen's inequality, possibly countering one upward bias with a downard one. For Poisson data, the variance function $V\left(\theta_{i}\right)=\theta_{i}$ is linear, and so $E\left[n V\left(\frac{1}{n} \bar{x}\right)\right]=n V(\mu)$ and the bias is not affected by the use of the variance function. This possibly explains why the inverted KR21 test performed worst for Bernoulli distributed observations and best for geometric or exponential distributed observations, but further research is needed.

\section{Conclusion and Discussion}

This paper has developed a methodology for deriving ANOVA hypothesis tests from reliability estimators. The generalized KR21 reliability estimators of Foster (2021) are inverted in this manner to obtain distribution-specific hypothesis tests for Bernoulli, Poisson, exponential, or geometric observations, shown in Table 1, under the assumption that the distribution of group means follows the corresponding conjugate prior. Under the same assumptions, Section 3.3 show that the standard ANOVA F-test statistic can be transformed into a consistent estimator of reliability. Section 4 shows that these inverted KR21 tests provide superior power over the standard ANOVA and GLM ANOVA without inflating the type I error rate for at least some scenarios.

The key to this methodology is the assumption that group means follow the conjugate prior for the corresponding exponential family distribution of observations. This assumption induces the linear posterior mean relationship between the group means and a hypothesized common mean necessary for a reliability of zero to be equivalent to complete shrinkage to the common mean. As with any assumption, it should be checked when possible, though it may not be possible when the number of groups $k$ is small. The results of Section 4, however, show that the inverted KR21 test may be robust to violation of this assumption. In any case, the assumption that group means follow the conjugate prior distribution, which is generally flexible, does not seem any more unreasonable than the near universal assumption of identical Gaussian errors.

The results of Section 4 indicate that the inverted KR21 test may in some scenarios provide increased power over standard ANOVA and GLM ANOVA without a corresponding increase in type I error rate, though the combinations of parameters considered was limited both by choice and necessity. There are almost certainly combinations of parameters which may produce results contradictory to the simulation study in Section 4. For example, preliminary studies conducted to determine parameter values for the larger simulation study in Section 4 indicated that if the first mean difference is taken to be $\mu-\delta$ rather than $\mu+\delta$, the generalized linear model ANOVA may strongly outperform the standard ANOVA and the inverted KR21 test. More research is needed into how the inverted KR21 test and how a generalized linear model ANOVA performs in different scenarios.

It should also be noted that this method does allow for differing group variances. For exponential family data, the variance is a function of the mean, as described in Section 
2. For non-normal exponential family data, all group means are equal if and only if all group variances are equal. This allows for the hypothesis test to function in the potential presence of differing group variances, as calculations made under the null hypothesis of equal group means are also calculations made under the hypothesis of equal group variances.

The transformation of the $\mathrm{F}$ test statistic which produces a reliability estimator, given in Equation (12), also raises an interesting possibility for post-test inference. If the null hypothesis of equal means is rejected, the estimated reliability $\hat{\rho}$ could be calculated from the $\mathrm{F}$ test statistic and then inserted into Equation (6) with $\bar{y}$ estimating $\mu$ to produce shrunken estimates of the means rather than using individual group means with a correction for multiple comparisons. Interval estimates could also be obtained easily by use of the fact that the estimate is a mean of a posterior distribution using a conjugate prior, whose properties should be known and should be converging to a normal distribution as the number of groups $k$ and observations $n$ increases. An estimate of the conjugate prior parameter $M$ can be obtained as $\hat{M}=n /(F-1)$, where $F$ is the $\mathrm{F}$ test statistic from a standard ANOVA. Bias correction may be necessary for any estimates. If multiple comparisons are performed with the shrunken estimates, then a correction to avoid inflating the false positive rate will still be required.

The inverted KR21 test of Section 3.2 relies on asymptotic normality by the central limit theorem to derive the $\chi_{k-1}^{2}$ reference distribution. For small number of observations $n$ within groups, it will not perform nominally. However, the results of Section 4 show that whatever the true reference distribution, it is close to the $F$ distribution when assuming normality. If the exact reference distribution could be derived using the properties of exponential families, it could provide distribution-specific ANOVA tests with nominal type I error rates in all situations.

Lastly, there also remains the possibility that other ANOVA tests corresponding to different models may be inverted into estimators of reliability, similar to the inversion of various ANOVA models into intraclass correlation coefficients in McGraw and Wong (1996), or that other reliability estimators may be inverted into ANOVA tests. It is possible that any reliability estimator may can be inverted into a corresponding ANOVA test for the same mode assumptions. The theory of reliability flourished initially with early connections to both Bayesian theory and ANOVA, and it is clear that there is still much fruitful research connecting the two to be uncovered in this area.

Acknowledgements: The author would like to thank his graduate school advisor Dr. Mark Kaiser who, though not directly involved in the production of this paper, guided and supported many of the ideas that would develop into this paper over conversations through the years, and whose influence runs deeply throughout. 
Difference in Power, Inverted KR21 ANOVA Minus Standard ANOVA, Bernoulli Data

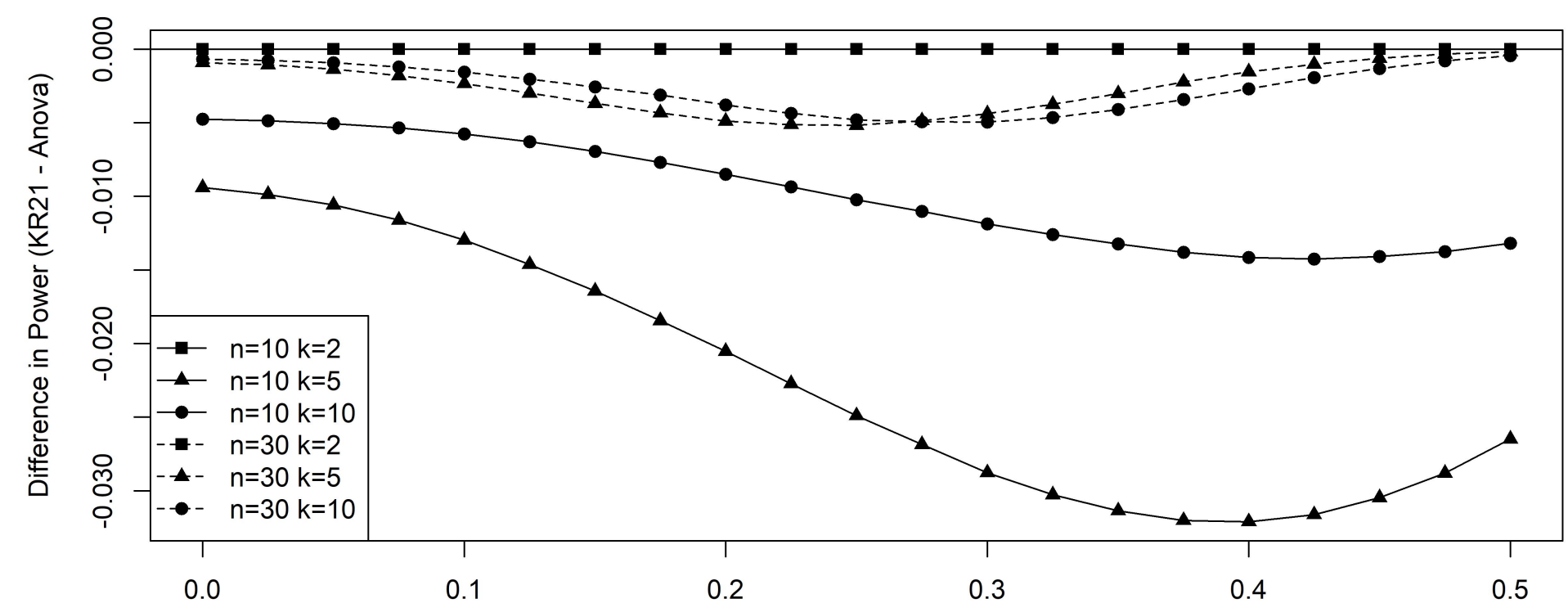

First Group Mean Difference $\delta$

Difference in Power, Inverted KR21 ANOVA Minus GLM ANOVA, Bernoulli Data

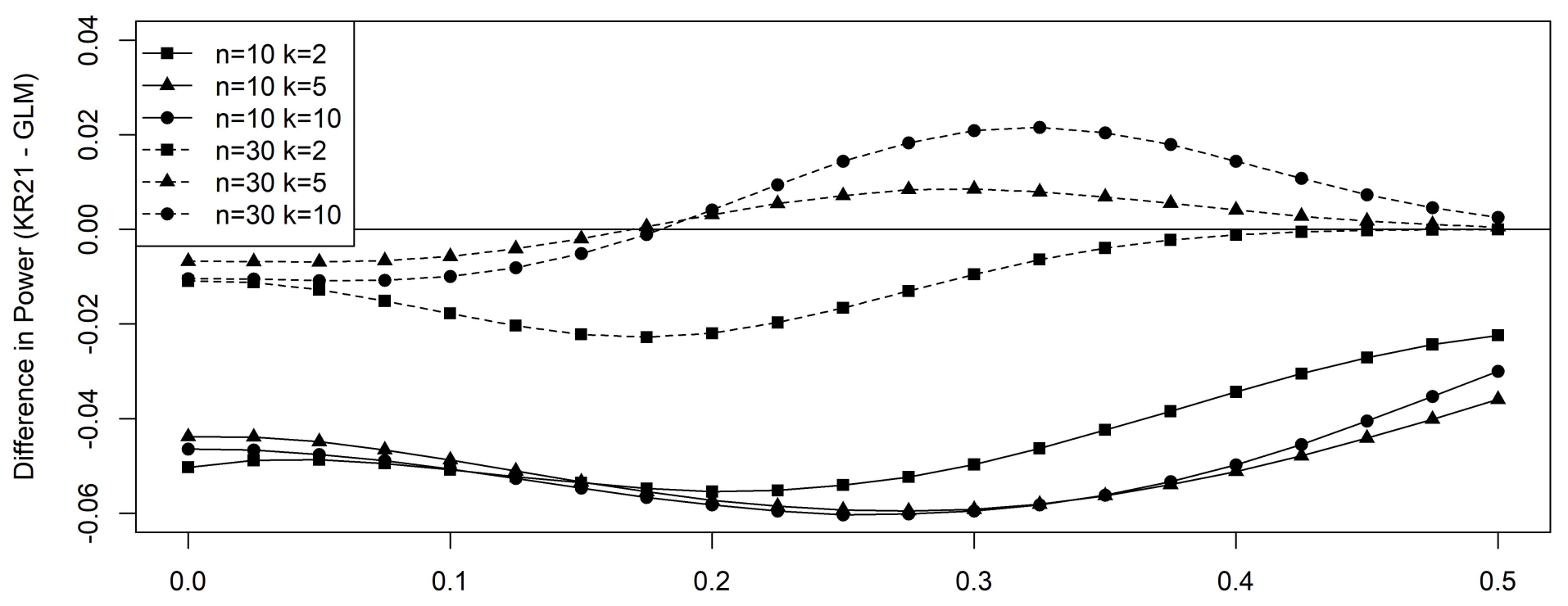

First Group Mean Difference $\delta$

Figure 1: A plot of the results of the simulation study described in Section 4 for Bernoulli distributed observations. The top plot shows the difference in power between the inverted KR21 test and the standard ANOVA and the bottom plot show the difference in power between the inverted KR21 test - GLM ANOVA. Both are plotted as a function of $\delta$, the amount added to the first group mean, for each $n$ and $k$. Selected exact results from this plot are given in Table 2 . 


\section{Difference in Power, Inverted KR21 ANOVA Minus Standard ANOVA, Poisson Data}

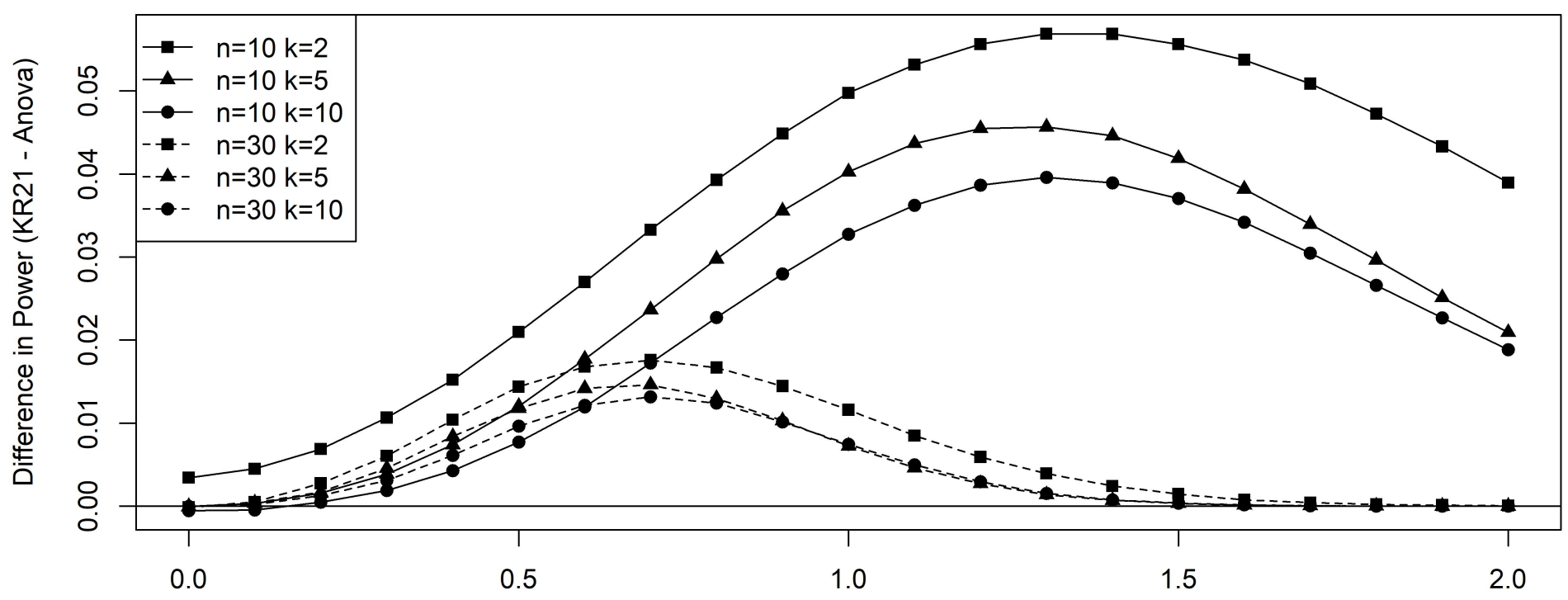

First Group Mean Difference $\delta$

Difference in Power, Inverted KR21 ANOVA Minus GLM ANOVA, Poisson Data

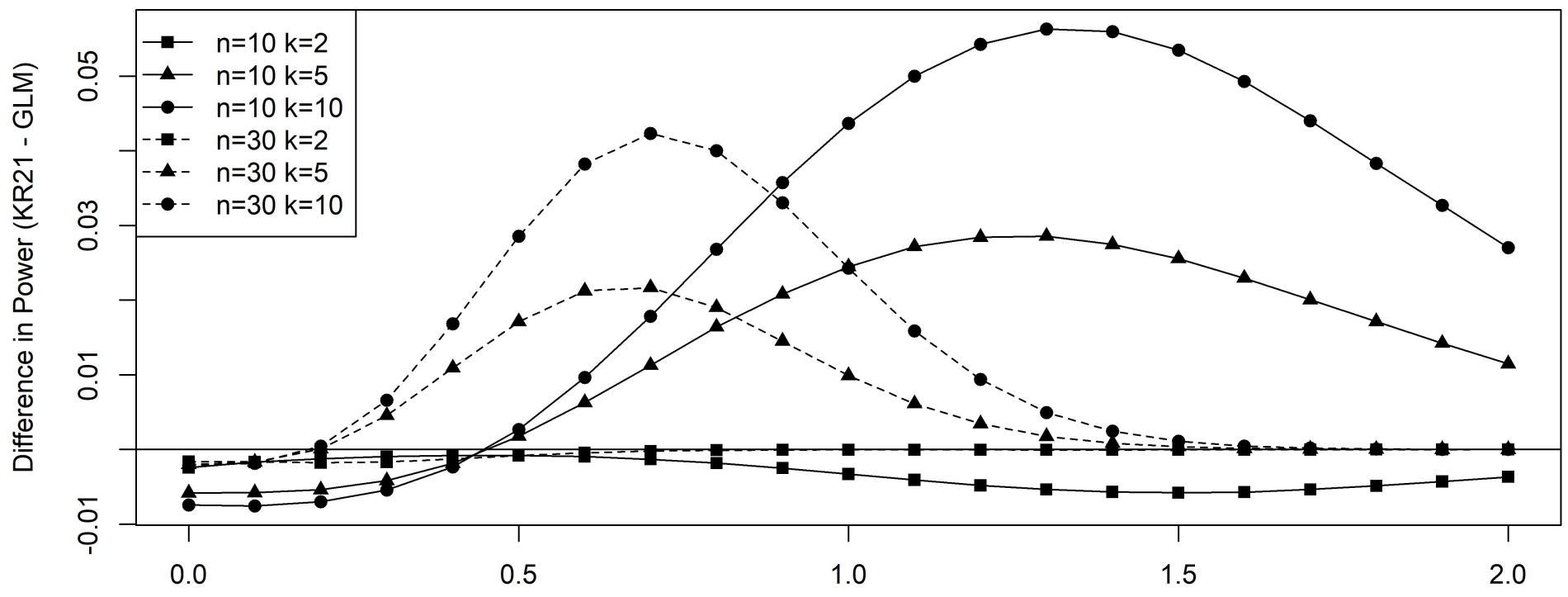

First Group Mean Difference $\delta$

Figure 2: A plot of the results of the simulation study described in Section 4 for Poisson distributed observations. The top plot shows the difference in power between the inverted KR21 test and the standard ANOVA and the bottom plot show the difference in power between the inverted KR21 test - GLM ANOVA. Both are plotted as a function of $\delta$, the amount added to the first group mean, for each $n$ and $k$. Selected exact results from this plot are given in Table 3 . 
Difference in Power, Inverted KR21 ANOVA Minus Standard ANOVA, Exponential Data

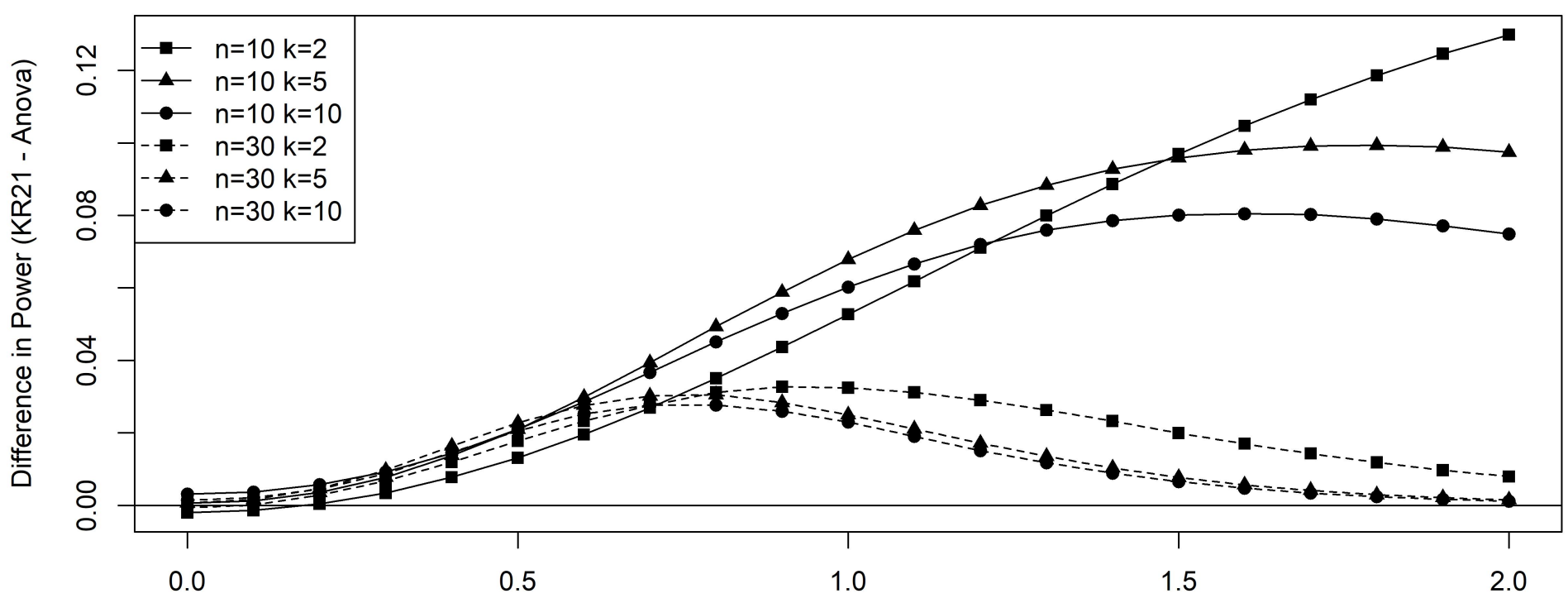

First Group Mean Difference $\delta$

Difference in Power, Inverted KR21 ANOVA Minus GLM ANOVA, Exponential Data

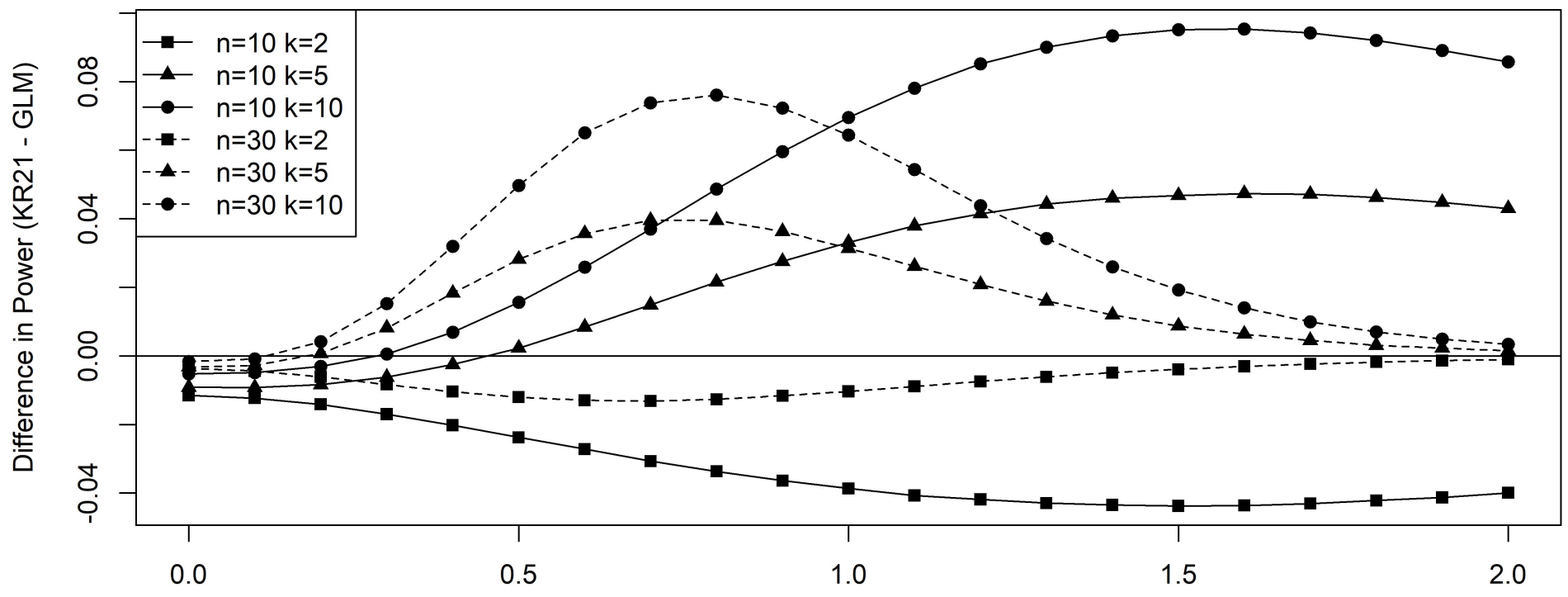

First Group Mean Difference $\delta$

Figure 3: A plot of the results of the simulation study described in Section 4 for exponential distributed observations. The top plot shows the difference in power between the inverted KR21 test and the standard ANOVA and the bottom plot show the difference in power between the inverted KR21 test - GLM ANOVA. Both are plotted as a function of $\delta$, the amount added to the first group mean, for each $n$ and $k$. Selected exact results from this plot are given in Table 4 . 
Difference in Power, Inverted KR21 ANOVA Minus Standard ANOVA, Geometric Data

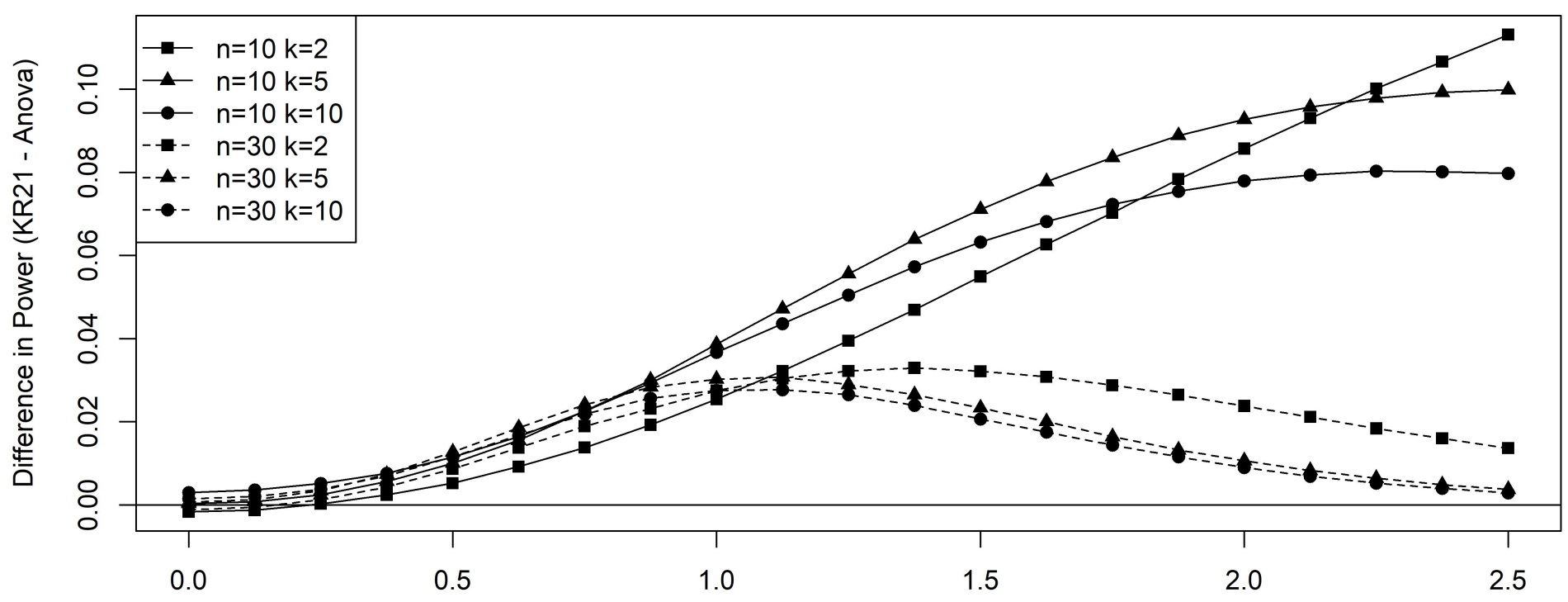

First Group Mean Difference $\delta$

Difference in Power, Inverted KR21 ANOVA Minus GLM ANOVA, Geometric Data

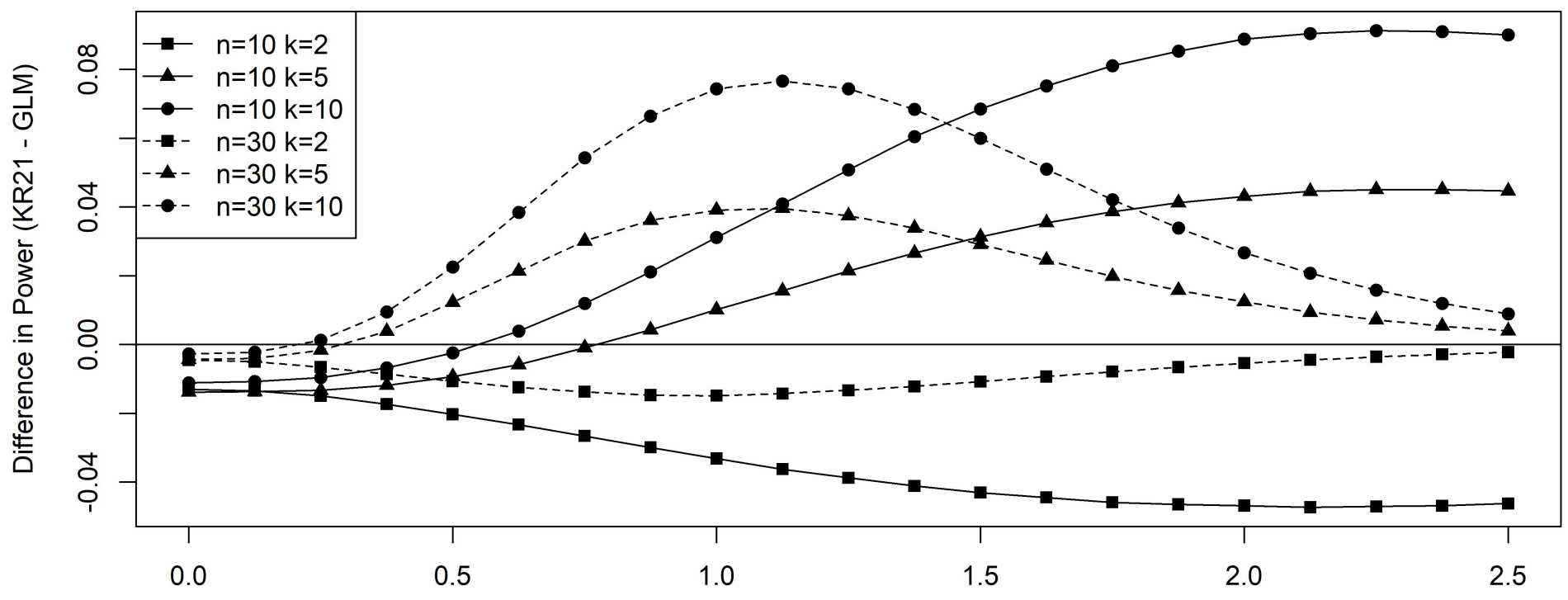

First Group Mean Difference $\delta$

Figure 4: A plot of the results of the simulation study described in Section 4 for geometric distributed observations. The top plot shows the difference in power between the inverted KR21 test and the standard ANOVA and the bottom plot show the difference in power between the inverted KR21 test - GLM ANOVA. Both are plotted as a function of $\delta$, the amount added to the first group mean, for each $n$ and $k$. Selected exact results from this plot are given in Table 5 . 


\begin{tabular}{|c|c|c|c|c|c|c|c|c|c|c|c|c|}
\hline & \multicolumn{4}{|c|}{$k=2$} & \multicolumn{4}{|c|}{$k=5$} & \multicolumn{4}{|c|}{$k=10$} \\
\hline \multirow{4}{*}{$n=$} & & KR21 & GLM & $\mathrm{OV}$ & & KR21 & GLM & $\mathrm{AOV}$ & & KR21 & GLM & $\mathrm{AOV}$ \\
\hline & & 0.035 & 0.085 & .035 & 0 & 0.042 & 0.086 & 0.052 & 0 & 0.045 & 0.092 & 0.050 \\
\hline & & 0.182 & 0.236 & 0.182 & & 0.183 & 0.243 & 0.208 & & 0.155 & 0.215 & 0.165 \\
\hline & & 0.619 & 0.641 & 0.619 & & 0.655 & 0.691 & 0.682 & & 0.593 & 0.623 & 0.606 \\
\hline \multirow{4}{*}{$n$} & & KR21 & GLM & $\mathrm{A}$ & & KR21 & GLM & & & & M & $\mathrm{OV}$ \\
\hline & & 0 & 0.060 & 0.049 & & 0.049 & 0.056 & 0. & & 0.048 & 0.059 & 0.049 \\
\hline & & 0.516 & 0.533 & 0.5 & & 0.536 & 0.529 & 0.541 & & 0.465 & 0.450 & 0.470 \\
\hline & $0-0.00$ & 0.985 & 0.985 & 0.985 & $\delta=0.50$ & 0.994 & 0.994 & 0.995 & $\delta=0.50$ & 0.991 & 0.989 & 0.992 \\
\hline
\end{tabular}

Table 2: Exact results for the simulation study described in Section 4 for Bernoulli distributed data. Each result is the power of the given test for the given combination of parameters. The three tests compared are the inverted KR21 test given in Table 1 (KR21), a generalized linear model ANOVA (GLM), and a standard ANOVA (AOV). Only results for $\delta=0, \delta=0.25$, and $\delta=0.50$ are shown.

\begin{tabular}{|c|c|c|c|c|c|c|c|c|c|c|c|c|}
\hline & \multicolumn{4}{|c|}{$k=2$} & \multicolumn{4}{|c|}{$k=5$} & \multicolumn{4}{|c|}{$k=10$} \\
\hline \multirow{4}{*}{$n=10$} & & KR21 & GLM & $\mathrm{AOV}$ & & KR21 & GLM & $\overline{\mathrm{AOV}}$ & & KR21 & GLM & $\overline{\mathrm{AOV}}$ \\
\hline & & 0.052 & 0.055 & 0.049 & 0 & 0.049 & 0.054 & 0.049 & $\delta=$ & 0.049 & 0.056 & 0.049 \\
\hline & & 0.458 & 0.461 & 0.408 & & 0.484 & 0.460 & 0.444 & & 0.436 & 0.392 & 0.403 \\
\hline & & 0.904 & 0.907 & 0.865 & & 0.950 & 0.938 & 0.929 & & 0.939 & 0.912 & 0.921 \\
\hline \multirow{4}{*}{$n=30$} & & $\mathrm{~K}$ & GLM & & & $\overline{K R}$ & $\mathrm{G}$ & & & & GLM & $\overline{\mathrm{AOV}}$ \\
\hline & & 049 & 1 & 0 & & 0.049 & 0.051 & 0.049 & & 0.049 & 0.052 & 0.050 \\
\hline & & 0.894 & 0.894 & 0.8 & & 0.935 & 0.925 & 0.928 & & 0.915 & 0.890 & 0.907 \\
\hline & $\delta=2.00$ & 1.000 & 1.000 & 1.000 & $\delta=2.00$ & 1.000 & 1.000 & 1.000 & $\delta=2.00$ & 1.000 & 1.000 & 1.000 \\
\hline
\end{tabular}

Table 3: Exact results for the simulation study described in Section 4 for Poisson distributed data. Each result is the power of the given test for the given combination of parameters. The three tests compared are the inverted KR21 test given in Table 1 (KR21), a generalized linear model ANOVA (GLM), and a standard ANOVA (AOV). Only results for $\delta=0, \delta=1$, and $\delta=2$ are shown. 


\begin{tabular}{|c|c|c|c|c|c|c|c|c|c|c|c|c|}
\hline & \multicolumn{4}{|c|}{$k=2$} & \multicolumn{4}{|c|}{$k=5$} & \multicolumn{4}{|c|}{$k=10$} \\
\hline \multirow{4}{*}{$n=10$} & & KR21 & GLM & $\mathrm{OV}$ & & KR21 & GLM & $\mathrm{OV}$ & & KR21 & GLM & $\mathrm{OOV}$ \\
\hline & & 0.041 & 0.053 & .043 & 0 & 0.045 & 0.054 & 0.044 & $\delta=$ & 0.050 & 0.055 & 0.047 \\
\hline & & 0.294 & 0.332 & 0.241 & & 0.379 & 0.346 & 0.311 & & 0.371 & 0.302 & 0.311 \\
\hline & & 0.637 & 0.677 & 0.507 & & 0.779 & 0.736 & 0.682 & & 0.782 & 0.696 & 0.707 \\
\hline \multirow{4}{*}{$n=30$} & & KR21 & GLM & $\mathrm{Ac}$ & & KR21 & GLM & $\mathrm{A}$ & & KR21 & GLM & $\mathrm{AOV}$ \\
\hline & & 0.04 & 0.051 & 8 & & 0.048 & 0.051 & 0.047 & & 0.050 & 0.052 & 0.049 \\
\hline & & 0.761 & 0.719 & 0.836 & & 0.836 & 0.804 & 0.8 & & 0.819 & 0.755 & 0.796 \\
\hline & 00 & 0.988 & 0.979 & 0.997 & $\delta=2.00$ & 0.997 & 0.996 & & $\delta=2.00$ & 0.997 & 0.993 & 0.996 \\
\hline
\end{tabular}

Table 4: Exact results for the simulation study described in Section 4 for exponential distributed data. Each result is the power of the given test for the given combination of parameters. The three tests compared are the inverted KR21 test given in Table 1 (KR21), a generalized linear model ANOVA (GLM), and a standard ANOVA (AOV). Only results for $\delta=0, \delta=1$, and $\delta=2$ are shown.

\begin{tabular}{|c|c|c|c|c|c|c|c|c|c|c|c|c|}
\hline & \multicolumn{4}{|c|}{$k=2$} & \multicolumn{4}{|c|}{$k=5$} & \multicolumn{4}{|c|}{$k=10$} \\
\hline \multirow{4}{*}{$n=10$} & & KR21 & GLM & $\mathrm{AOV}$ & & KR21 & GLM & $\overline{\mathrm{AOV}}$ & & KR21 & GLM & AOV \\
\hline & & 0.041 & 0.054 & 0.043 & 0 & 0.044 & 0.058 & 0.044 & $\delta=$ & 0.050 & 0.061 & 0.047 \\
\hline & & 0.240 & 0.278 & 0.200 & & 0.312 & 0.291 & 0.257 & & 0.306 & 0.255 & 0.256 \\
\hline & & 0.550 & 0.596 & 0.436 & & 0.702 & 0.657 & 0.602 & & 0.705 & 0.615 & 0.625 \\
\hline \multirow{4}{*}{$n=30$} & & KR2 & GLM & & & & $\mathrm{G}$ & & & & GLM & AOV \\
\hline & & .047 & .051 & 8 & & 0.048 & 0.052 & 0.047 & & 0.050 & 0.053 & 0.048 \\
\hline & & 0.662 & 0.676 & 0.6 & & 0.752 & 0.714 & 0.723 & & 0.730 & 0.655 & 0.703 \\
\hline & $\delta=2.50$ & 0.970 & 0.972 & 0.956 & $\delta=2.50$ & 0.991 & 0.987 & 0.987 & $\delta=2.50$ & 0.990 & 0.982 & 0.988 \\
\hline
\end{tabular}

Table 5: Exact results for the simulation study described in Section 4 for geometric distributed data. Each result is the power of the given test for the given combination of parameters. The three tests compared are the inverted KR21 test given in Table 1 (KR21), a generalized linear model ANOVA (GLM), and a standard ANOVA (AOV). Only results for $\delta=0, \delta=1.25$, and $\delta=2.5$ are shown. 


\section{References}

Allison, P. D. (1978). The reliability of variables measured as the number of events in an interval of time. Sociological Methodology, 9, $238-253$.

Blanca, M., Alarcón, R., Arnau, J., Bono, R., \& Bendayan, R. (2017, 10). Non-normal data: Is anova still a valid option? Psicothema, 29, 552-557.

Bono, R., Blanca, M. J., Arnau, J., \& Gómez-Benito, J. (2017). Non-normal distributions commonly used in health, education, and social sciences: A systematic review. Frontiers in Psychology, 8, 1602.

Casella, G. (1985). An introduction to empirical bayes data analysis. The American Statistician, 39(2), 83-87.

Casella, G., \& Berger, R. (2002). Statistical inference, second edition. Duxbury Press.

Cronbach, L. J. (1951, September). Coefficient alpha and the internal structure of tests. Psychometrika, $16(3), 297-334$.

Diaconis, P., \& Ylvisaker, D. (1979). Conjugate priors for exponential families. The Annals of Statistics, 7(2), $269-281$.

Feldt, L. S. (1965, September). The approximate sampling distribution of kuderrichardson reliability coefficient twenty. Psychometrika, 30(3), $357-370$.

Fisher, R. A. (1925). Statistical methods for research workers. In (11th ed.). Edinburgh.

Foster, R. C. (2020, June). A generalized framework for classical test theory. The Journal of Mathematical Psychology, 96.

Foster, R. C. (2021, February). Kr20 and kr21 for some nondichotomous data (it's not just cronbach's alpha). Educational and Psychological Measurement.

Gelman, A., \& Hill, J. (2006). Data analysis using regression and multilevel/hierarchical models. Cambridge Universty Press.

Hoyt, C. J. (1941). Test reliability estimated by analysis of variance. Psychometrika, $6,153-160$.

Keats, J. A. (1964, September). Some generalizations of a theoretical distribution of mental test scores. Psychometrika, 29(3), 215 - 231.

Kelley, T. (1923). Statistical method. New York: Macmillan.

Knief, U., \& Forstmeier, W. (2018). Violating the normality assumption may be the lesser of two evils. Behavior Research Methods.

Kuder, G. F., \& Richardson, M. W. (1937). The theory of the estimation of test reliability. Psychometrika, 2(3), $151-160$.

Lantz, B. (2013). The impact of sample non-normality on anova and alternative methods. British Journal of Mathematical and Statistical Psychology, 66(2), 224-244.

Levy, R., \& Mislevy, R. J. (2016). Bayesian psychometric modeling. Chapman \& Hall/CRC.

Lord, F. M., Novick, M. R., \& Birnbaum, A. (1968). Statistical theories of mental 
test scores. Oxford, England: Addison-Wesley.

McGraw, K. O., \& Wong, S. P. (1996). Forming inferences about some intraclass correlation coefficients. Psychological methods, 1(1), 30.

Morris, C. N. (1982). Natural exponential families with quadratic variance functions. The Annals of Statistics, 10(1), $65-80$.

Morris, C. N. (1983). Natural exponential families with quadratic variance functions: Statistical theory. The Annals of Statistics, 11(2), 515-529.

Nelder, J. A., \& Wedderburn, R. W. M. (1972). Generalized linear models. Journal of the Royal Statistical Society: Series A (General), 135(3), 370-384.

Novick, M. R. (1969). Multiparameter bayesian indifference procedures. Journal of the Royal Statistical Socity. Series B (Methodological), 31(1), 29-64.

Novick, M. R., Jackson, P. H., \& Thayer, D. T. (1971, September). Bayesian inference and the classical test theory model: Reliability and true scores. Psychometrika, $36(3), 261-288$.

Raykov, T., \& Marcoulides, G. A. (2019). Thanks coefficient alpha, we still need you! Educational and Psychological Measurement, 79(1), 200-210.

Zimmerman, D. W., \& Zumbo, B. D. (2015). Resolving the issue of how reliability is related to statistical power: Adhering to mathematical definitions. Journal of Applied Modern Statistical Methods, 14(5). 